\title{
Frequency bandwidth enhancement using continuous wavelet transform and random noise attenuation by f-xy filtering technique - a case study from Badin oilfield, Indus basin, Pakistan
}

\author{
Asad Taimur ${ }^{1}$, Akinniyi Akinsunmade ${ }^{1}$, Sylwia Tomecka-Suchon ${ }^{1,{ }^{*}}$ and Fahad Mehmood ${ }^{2}$ \\ ${ }^{1}$ Department of Geology, Geophysics and Environmental Protection AGH University of Science and \\ Technology, Krakow, Poland \\ ${ }^{2}$ Department of Earth and Environmental Sciences, Bahria University, Islamabad, Pakistan
}

\begin{abstract}
Routine seismic data processing does not always meet the quantitative interpreters' expectations especially in areas like Badin, where prospective thin bed $\mathrm{B}$ - sand interval is ambiguous throughout the seismic volume. Continuous Wavelet Transform (CWT) provides detailed description of seismic signal in both time and frequency without compromising on window length and a fixed time-frequency resolution over time-frequency spectrum. We present enhancement of seismic data for effective interpretation using the bandwidth extension technique. Implementing bandwidth extension, the dominant frequency increases from $18 \mathrm{~Hz}$ to $30 \mathrm{~Hz}$ and the frequency content boosted from $40 \mathrm{~Hz}$ to 60 Hz. Noise inclusion by the technique was suppressed by F-XY predictive filter and F-XY deconvolution with edge preserve smoothing. Phase and spectral balancing were applied to partial angle stacks to stabilize the phase rotation across the 3D survey, particularly for far offset stack. Frequency was balanced using surface consistent spectrum balancing, and subjected to trace scaling for amplitudes balance and preservation. Results of the techniques yielded unique improvement on the data resolution and subtle information about the thin sand beds were better delineated. Tuning thickness analysis reveals the usefulness of bandwidth extension, with an increase of $30 \%$ in the resolving power of thin beds.
\end{abstract}

Keywords: CWT, 3D F-XY filtering, Bandwidth extension, tuning thickness analysis

\section{Introduction}

Seismic data pre-conditioning was performed on a portion of $3 \mathrm{D}$ volume from Badin area dominated by random noise and slightly poor resolution. Such datasets are not considered to be suitable for impedance inversion and could lead to uncertainty in reservoir prediction

\footnotetext{
*Corresponding author: tomecka@agh.edu.pl
} 
[1]. Furthermore, the reflector continuity was not good to be correlated along the inlines and crosslines. The pre-conditioning of the seismic data showed a remarkable improvement in the continuity of the reflectors, recognition of small faults, improvement in the frequency bandwidth of the data and harmonizing the phase as well as amplitude of the seismic data. This process was applied to both partial and full stack data. The flow chart of the seismic data pre-conditioning is illustrated in (Fig. 1).

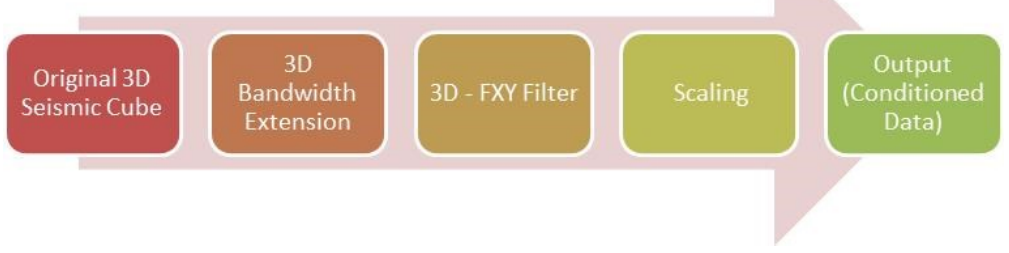

Fig. 1. Workflow for seismic data pre-conditioning.

\subsection{D Bandwidth extension}

Propagating seismic waves in the subsurface lose frequency content with depth. The higher frequency content gets attenuated more rapidly as compared to low frequency content and is received on the geophones with narrow frequency spectrum [2]. The enhancement of frequency bandwidth results in the increase of seismic resolution and the reservoir structure and architecture are more clearly defined as both are less affected by low frequency noise [3]. The increase in the seismic frequency bandwidth results in the removal of noise and the enhancement of seismic resolution.

The seismic wavelet contains embedded information about subsurface reflectors. The higher frequency content can be restored through digital seismic data processing. There are several methods in seismic data processing to increase the frequency content like spiking deconvolution and spectral whitening [4]; loop deconvolution [5]. All of these methods tend to increase the higher side of frequency bandwidth spectrum, but it also tends to increase the noises. Much effective technique was applied in this study, which extends the signal and frequency both at the higher and the lower ends and which also maintain a balance of the frequency spectrum across the 3D survey. This technique is known as Continuous Wavelet Transform (CWT) [6]. The continuous wavelet transform is followed by the application of F-XY deconvolution filters, which remove the noise with the preservation of edges.

\subsection{Continuous Wavelet Transform (CWT)}

The seismic trace in the frequency domain can be analyzed conventionally using the Fast Fourier Transform (FFT). One of the problems associated with the Fast Fourier Transform is that it transforms the local information into global information. The Short Fourier Transform on the other hand helps preserving the local information. However the short window chosen in case of Short Fourier Transform constrains the frequency and the results depends upon the length of the window chosen. Consequently, the Continuous Wavelet Transform does not have the same restriction and it can possess both the local and global information.

Continuous Wavelet Transform (CWT) is however used to apply the time series analysis of a seismic trace, which in fact decomposes the seismic trace in frequency and phase in frequency and time domain. It is basically a time series $x(t)$ convolved with scaled 
(b) and translated (a) wavelet $\Psi(\mathrm{t})$. The scaled wavelets are known as the daughter wavelets which are represented by equation 1 .

$$
X_{w}(a, b)=\frac{1}{|a|} \int_{-\infty}^{\infty} x(t) \bar{\psi}\left(\frac{t-b}{a}\right) d t
$$

Continuous Wavelet Transform in its true sense is a discrete operator rather than a continuous operator. Therefore a choice needs to be brought in for how many daughter wavelets would be used. Moreover the mother wavelet must also meet the acceptability condition, as the studying wavelet would be used to rebuild the original time series after the frequency bandwidth is extended. Therefore, the Morlet Wavelet is used as mother wavelet. The Morlet Wavelet is actually a complex function that represents a plane wave tempered by Gaussian Function. The complex nature of Morlet Wavelet allows the computation of phase and amplitude for each scale at distinct times. Continuous Wavelet Transform provides a very fine and detailed description of seismic signal in terms of both time and frequency. Both these properties are used to predict the harmonics and sub-harmonics which are used for frequency bandwidth extension.

The computation of harmonics and sub-harmonics of limited seismic data could be carried out using the time series analysis of Continuous Wavelet Transform (CWT). The harmonics is basically an integer multiple of a central frequency and sub-harmonics a quotient over half of an integer multiple. In this study the complex mother wavelet has been used and phase and amplitude spectrum was available for this computation. By convolving the harmonics and sub-harmonic frequencies with the seismic trace, the required information and the reflectivity could be disclosed, however, it was difficult to detect previously. The recovering of the reflectivity is basically accomplished by the extension of frequency bandwidth, adding the harmonics and the sub-harmonic frequencies to the seismic trace. This whole process is like convolution as the harmonics and sub-harmonic frequencies are added to the seismic trace. As such it refers to adding the reflectivity to the seismic trace [7]. The last step involved is the renovation of the altered time series from CWT domain of time and scale to the newly formed seismic trace with the extended frequency bandwidth formed after this whole procedure.

The fundamental frequency for computing harmonics and sub-harmonics are user defined. This frequency is chosen after analyzing the seismic spectrum. An octave lying below is used for computing harmonic while octave lying above for sub-harmonics frequency and scales. The harmonic/sub-harmonic can increase and decrease octaves, which obviously honor the Nyquist and zero hertz boundaries..

\section{Methodology}

\subsection{Bandwidth extension technique}

The frequency bandwidth extension technique was applied to the 3D seismic cube of the Badin study area in Lower Indus Basin, Sindh, Pakistan. The seismic data of this area was generally in good quality with moderate signal to noise ratio and relatively weak seismic resolution suppressed by random noise. The frequency parameters applied to the 3D seismic data have start to end frequency of 8-45 Hertz which remains the same as that of original data and extension end frequency of 90 Hertz has been applied with $3 \times 3$ of inline / crossline and 250 millisecond time window. 


\subsection{F-XY Prediction filtering}

In seismic data processing the prediction filtering is one of the most impressive techniques to attenuate noise; a technique is also known as F-XY deconvolution or F-XY prediction. In this technique the signal is predicted by the nearby trace and noise is an unpredicted aspect. After stacking the noises present in the seismic data results in incoherent or random noises. The F-XY deconvolution works on each frequency slice in the frequency-spatial domain [8]. F-XY deconvolution calculates a two dimensional two sided complex Wiener Prediction Filter for each Mono-frequency series. This filter is then applied, which calculates the inverse F-XY transform. F-XY deconvolution can also be used for frequency interpolation. The main advantage of using $3 \mathrm{D}$ filter over $2 \mathrm{D}$ is to produce less distortion in geology [9].

The time slice is converted into a frequency slice, when the seismic data is converted from time and space $(x, y)$ to frequency and space $(x, y)$. In the converted data, each sample has both real and imaginary constituents. The events having the similar spatial dips appear as a sinusoidally complex signal along a given frequency slice which can be described by the following equation as equation 2

$$
(\cos w(a x+b y)-i \sin (a x+b y))
$$

This process improves resolution and which in turn enhances the signal. The F-XY prediction filtering process works well using a complex rectangular filter to calculate the signal at the central point of the spatial filter. If there is any dissimilarity between the predicted complex value and the actual value, it can be then categorized as noise and hence removed from the data. The time volume can also be optionally divided into small windows to have better approximate the conjecture of constant three-dimensional dips.

\subsection{Other data processing Applied}

In this research work, both the phase and spectral balancing was also applied to partial angle stacks, in order to stabilize the phase across the 3D survey mostly for far stack. Using the surface consistent spectrum balancing, the frequency is also balanced. Further the trace scaling is applied to the data to balance the amplitudes and it is also ensured that the original amplitudes remain preserved.

\section{Results and Discussion}

\subsection{Implementation of 3D Bandwidth extension}

Figure $2 \mathrm{a}$ shows the original seismic section and figure $2 \mathrm{~b}$ the seismic section with enhanced frequency spectrum after the application of technique bandwidth extension. Figure $2 \mathrm{a}$ and figure $2 \mathrm{~b}$ display the original seismic section before applying the frequency bandwidth extension and after applying the frequency bandwidth extension respectively. As observed from the above figures, it shows that extending the seismic frequency bandwidth has resulted in sharpening of the wavelet, which thereby attenuate wavelet compression and increase in temporal resolution. It can be clearly seen from the plotted frequency spectrum that the peak frequency content has extended from $18 \mathrm{~Hz}$ to $30 \mathrm{~Hz}$. Also the frequency content is boosted at the higher end as compared to the original bandwidth from $40 \mathrm{~Hz}$ to $60 \mathrm{~Hz}$. The frequency content has increased, but consequently as discussed above it also has enhanced the noises for which removal technique has been discussed in the following section. It is obviously seen from the displayed sections (Fig. 2a and 2b), that $a$ 
considerable improvement in spatial resolution of the data is an observed phenomena with comparison of the figures.

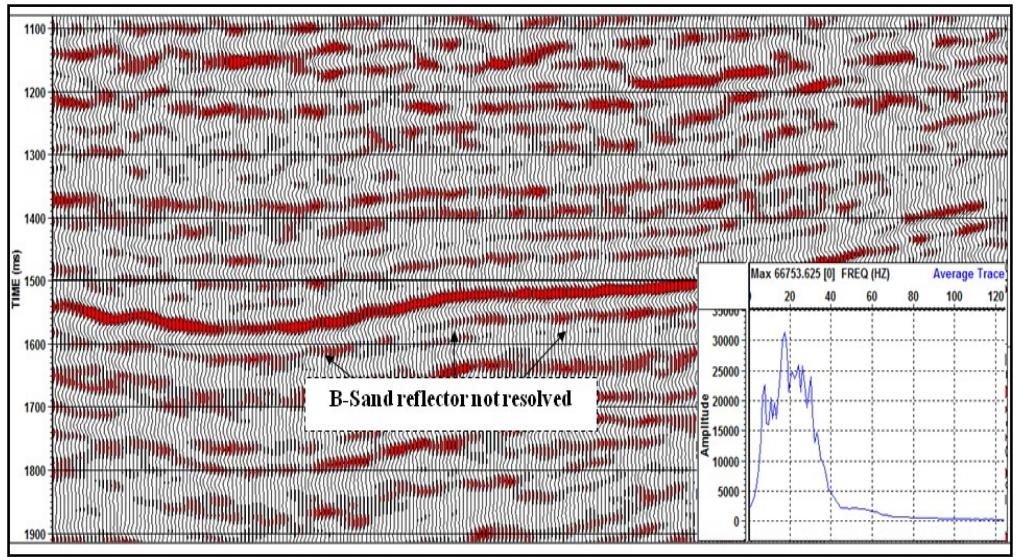

Fig. 2a. Original inline 7768 from 3D seismic cube, before applying frequency bandwidth extension to the seismic data.

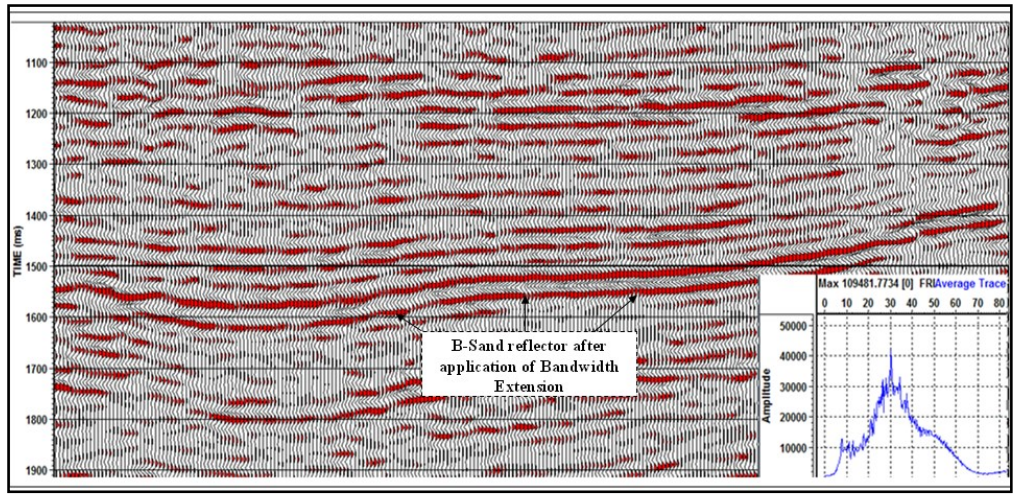

Fig 2b. Inline 7768 from 3D seismic cube, after applying frequency bandwidth extension to the seismic data. The B-sand reflector gets clearly resolved.

\subsection{F-XY prediction filtering}

The original 3D seismic cube was slightly noisy. After the application of frequency bandwidth extension the frequency as well as signal to noise ratio was improved to a considerable extent but noises were also enhanced. These noises can be removed by the application of F-XY deconvolution to the data. When F-XY deconvolution is applied to the 3D seismic cube of Badin study area, it has done a tremendous job by removing random noise from the data with edge preserve smoothing (Fig. 3).

Figure 4 shows the comparison of original and enhanced data after the application of FXY deconvolution, on dip section along inline-7969. The circled areas and rectangle in the top half show the shabby appearance and discontinuity of the reflectors, however, in the bottom half they have come up in much better resolution, continuity and clarity of small faults after applying the 3D FXY prediction filtering and F-XY deconvolution. 


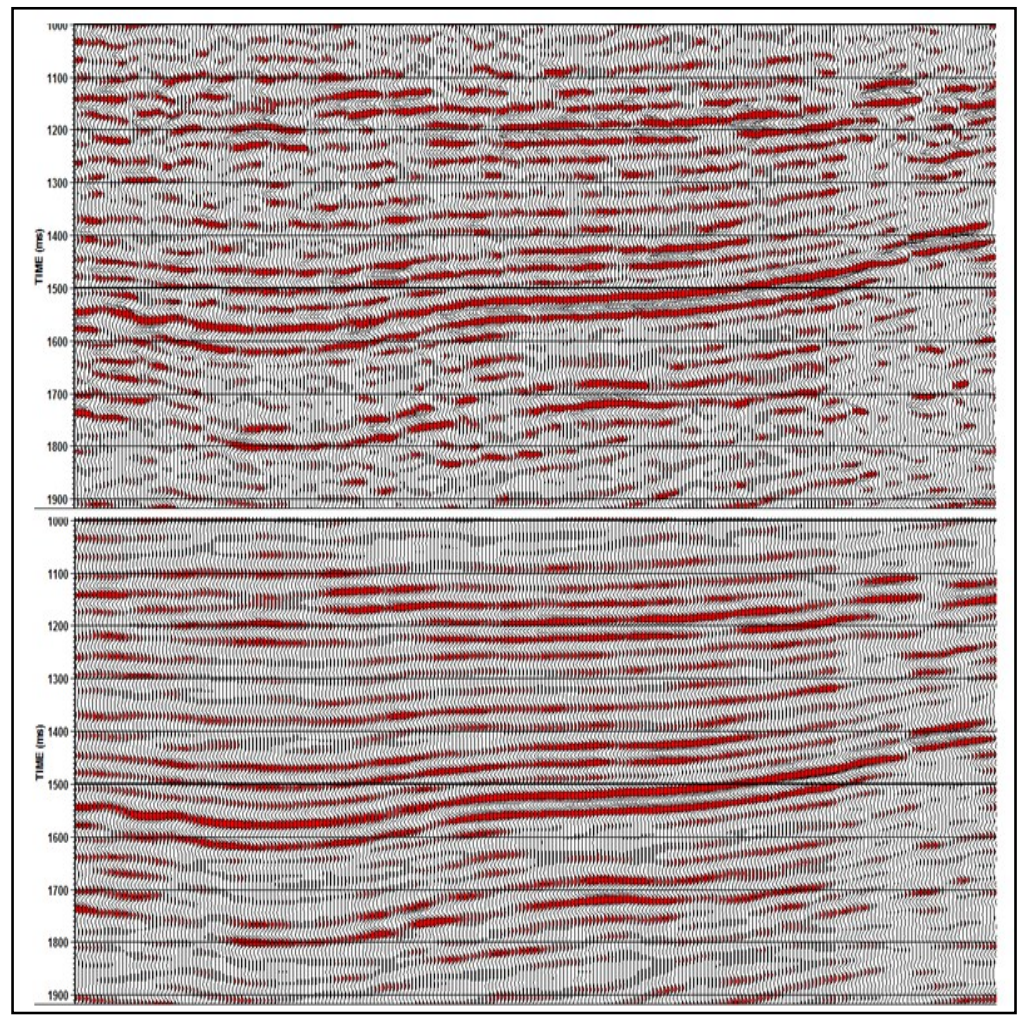

Fig. 3. (Top) the 3D seismic inline after the application of frequency bandwidth extension. (Bottom) same seismic inline after the application of $3 \mathrm{D}$ prediction filter.

Figure 5 is a time slice of the horizon B sand of the cretaceous age which is a major producer of hydrocarbons in the Badin area, but has low resolution and gets disappeared as various places on the original dataset (top). After the application of the above mentioned process, this horizon gets visible as marked by an arrow on time slice and clearly reflects the reduction in the amount of noises and the appearance and sharpness of the reflectors is quite evident (bottom).

\subsection{F-XY Tuning Thickness Analysis}

The tuning thickness analysis has been carried out to analyze what difference the bandwidth extension has done to the 3D seismic data. (Fig. 6a) shows the tuning thickness analysis between the old wavelet and the new wavelet chosen for bandwidth extension. It can be observed that for the old wavelet, the normalized peak-trough amplitude at actual time thickness of $0.022 \mathrm{sec}$, reaches maximum amplitude which is nearly 1.2 at the tuning thickness. Later it tends to start decreasing with decreasing tuning thickness and has maximum resolving power of 0.022 seconds. Consequently, (Fig. 6b) shows the new wavelet, which reaches the maximum amplitude of 1.6 at tuning thickness of 0.015 seconds with a resolving power of thin bed up to 0.015 seconds. Hence it can be observed that the power of resolving thin beds has increased tremendously approximately up to $32 \%$ as compared to the original seismic data. 


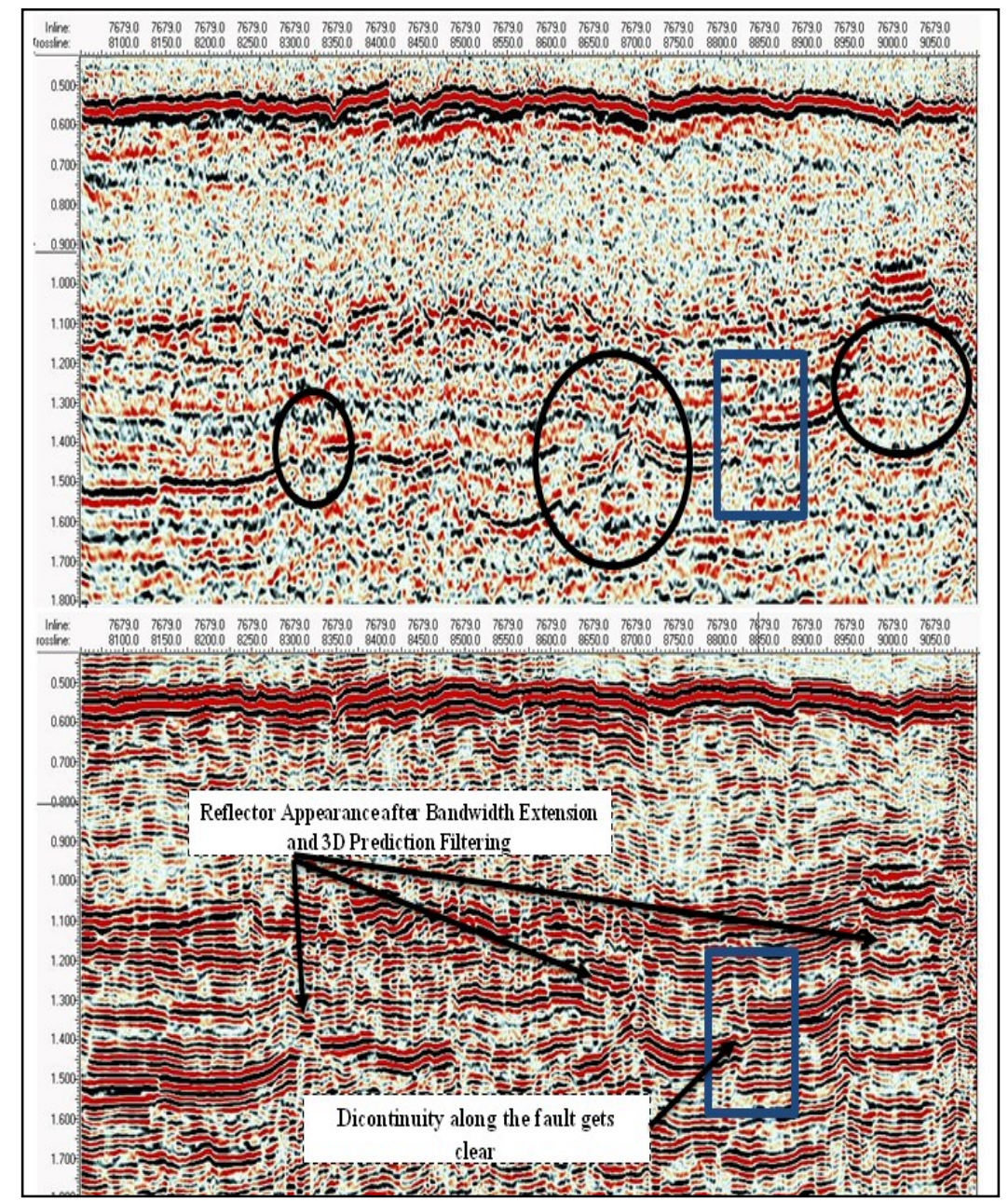

Fig. 4. Original 3D seismic inline 7679 before the application of Frequency Bandwidth Extension and F-XY Prediction Filtering (Top) and same seismic inline after the application of Frequency Bandwidth Extension and F-XY Prediction Filtering.

The formula for vertical resolution for seismic data can be given as $\mathrm{v}=\mathrm{f} \lambda$, where $\lambda / 4$ is the tuning thickness for seismic. The velocity analysis of the study area has built up a hypothesis that the interval velocity at reservoir level of interest is approximately $3500 \mathrm{~m} / \mathrm{s}$, and the dominant seismic frequency for the old data was $18 \mathrm{~Hz}$ and for the new data after bandwidth extension was $30 \mathrm{~Hz}$. With this hypothesis, the minimum thickness of 49 meters seismic can resolve for old data and for new data with inculcation of the bandwidth extension it can now resolve seismic for a thinner bed of 29 meters. A quite good improvement has therefore resulted in towards resolving power of seismic data, and improved resolution also facilitates in seismic to well tie process [10]. 


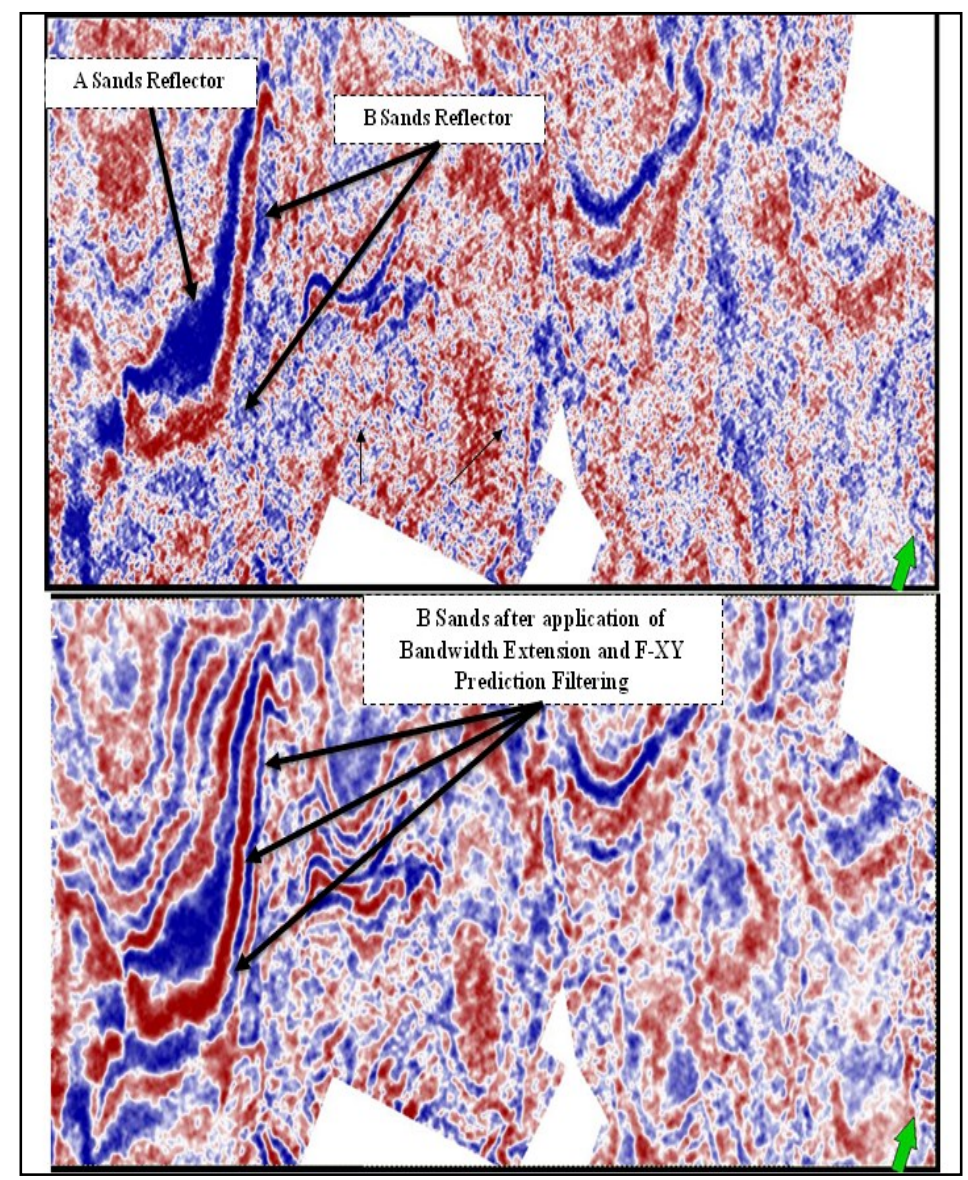

Fig. 5. Comparison of time slice at 1500 millisecond from original 3D seismic cube (Top) and same time slice after the application of Frequency Bandwidth Extension and F-XY Prediction Filtering (Bottom).

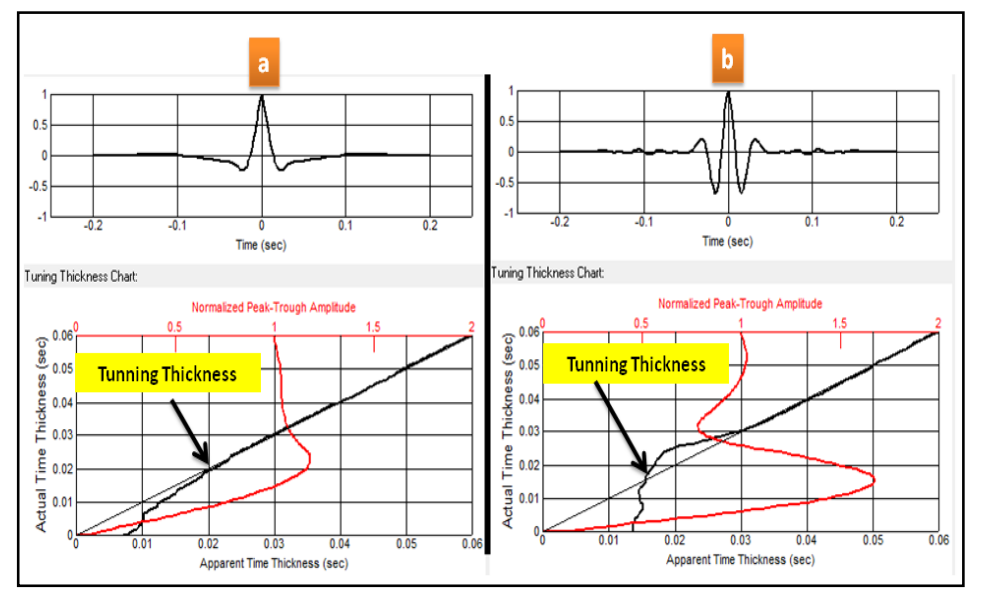

Fig. 6. a) Old wavelet; b) new wavelet extracted from the seismic data for interval of 0-2000 milliseconds with tuning thickness. 
In old data issues related to the marking of B sands have come up in the main reservoir in Badin study area. It is also observed that B sands tends to get thicker towards the west approximately up to 50 meters above the tuning thickness but can be resolved easily and marked as peak on seismic. However, towards east, the thickness of B sands gets thinner approximately 30 meters below the tuning thickness and marked as trough. After Bandwidth extension, the B sands can be resolved easily and marked as peak throughout the $3 \mathrm{D}$ seismic cube, as new data has now the resolving power of approximately 29 meters.

\section{Conclusion}

The adequate preconditioning of seismic data applied in has impressively enhance the resolution which is adequate and comprehensive to perform seismic inversion studies and is also equally effective in improved structural interpretation. The temporal resolution of seismic data is increased with $3 \mathrm{D}$ bandwidth extension and continuous wavelet transform algorithm without boosting any additional noise. The peak frequency of data was improved from $18 \mathrm{~Hz}$ to $30 \mathrm{~Hz}$ with suitably boosting the frequencies at higher end of spectrum as well. There was an improvement of $30 \%$ in resolving power of beds in new data. The use of structure oriented filtering with edge preserve smoothing that runs simultaneously on inline and crossline. Besides, it improves lateral and vertical resolution in the target area removing unwanted noises and balancing the amplitude content along the seismic record. This methodology applied to both post and pre-stack seismic data results in clean seismic volumes with improved frequencies and resolution suitable for seismic interpretation and ready to be inverted.

Paper was financially supported from the research subsidy nr 16.16.140.315 at the Faculty of Geology Geophysics and Environmental Protection of the AGH University of Science and Technology, Krakow, Poland, 2019.

\section{References}

1. R.B Latimer, R. Davison, P. van Riel, Lead. Edge. 19,3 (2000)

2. P.G. Richards, C.W. Frasier, Geophysics. 41, 441-458 (1976)

3. F. Gassmann, Geophysics. 16 673-685 (1951)

4. S. Kumar, K. Kumari, A. Biswal, Canadian Society of Exploration Geophysicists Recorder, 10 (2008)

5. P. Young, A. Wild, CSEG National Convention, (2005)

6. M. Smith, G. Perry, J. Stien, A. Bertrand, A. Yu, First break 26, 97-102 (2008)

7. R. Simm, M. Bacon,. Handbook, University Press, Cambridge. 279p.(2014)

8. J.A. Cadzow, IEEE Trans. On Acoustics, Speech, and Signal Processing 36 49-62 (1988)

9. M.K. Chase, Geophysics 23, 51-56 (1992)

10. R.K. Shrestha, Society of Exploration Geophysicists Spring Symposium Technical Program 11, 5-16. (2008) 